\title{
In-situ Characterization of Catalytic Reactions Promoted by Localized Surface Plasmon Resonance Energy
}

\author{
Canhui Wang ${ }^{1,2}$, Wei-Chang Yang ${ }^{1,2}$, Devika Sil ${ }^{1,2}$, Amit Agrawal ${ }^{1,2}$, and Renu Sharma ${ }^{1 *}$. \\ 1. Center for Nanoscale Science and Technology, National Institute of Standards and Technology, \\ Gaithersburg, USA. \\ 2. Maryland NanoCenter, University of Maryland, College Park, USA.
}

Local surface plasmon resonances (LSPRs) are generated in nanoparticles of certain metals such as Au, $\mathrm{Ag}$, and $\mathrm{Al}$ due to the interaction of photons or electrons with the metal free electrons. Recently, optical methods have been used to show that 'hot electrons' generated from the decay of surface plasmons can trigger chemical reactions at room temperature. [1-2] Understanding the reactions promoted by LSPR is important for designing efficient catalytic systems for a wide range of energy and environmental applications. However, many important questions related to such reaction processes remain unanswered due to the complexity of the reaction kinetics, and the limited spatial resolution of current optical methods. The complexity arises from the dynamic interplay between the nanoparticle and the reactive environment at the nanoscale. For instance, gas absorption on the surfaces of the nanoparticles affects the free energy of the exposed surfaces and can lead to a morphological changes or induce surface oscillations in nanoparticles. [3-4] Since the LSPR is known to strongly depend on the size, shape and dielectric environment of the particles, any change in one or more of these parameters will also affect the spatial distribution of intensity and energy of the LSPR. Therefore, one of the fundamental questions that needs to be answered is how changes in the particle morphology and/or the dielectric environment during photocatalytic reactions affect the spatial distribution of LSPR profiles and if that can be correlated with the location of each reaction step.

We use an ensemble of techniques to characterize LSPR-promoted chemical reactions, at high resolution, in an environmental transmission electron microscope equipped with a monochromated electron source, which yields an energy resolution of $80 \mathrm{meV}$. In particular, we focus on the LSPRpromoted dissociation of $\mathrm{H}_{2}$ gas near aluminum nanoparticles. Electron energy-loss spectra (EELS) imaging, with different energy dispersions, is used to acquire both elemental $(0.25 \mathrm{eV}$ dispersion) and LSPR maps (0.01 eV dispersion) from the same particle. These combined spectrum images allow correlation of the LSPR profile and the morphology of different compounds in the nanoparticle (Fig. 1), providing insight into particle engineering for optimum LSPR output. The result shows that the LSPR intensity distribution is determined by the shape of the $\mathrm{Al}$ core, rather than the particle exterior (see Fig. 1A-1B), while the LSPR energy distribution depends on the $\mathrm{Al}_{2} \mathrm{O}_{3}$ shell thickness (Fig. 1C-1F). In a $100 \mathrm{~Pa} \mathrm{H}_{2}$ gaseous environment we observe oscillatory morphological changes in the core, while the shell remains relatively unchanged, showing the effect of hydrogen interaction with the core under electron beam irradiation. Comparison of LSPR locations and shifts between the EELS maps, before and after gas exposure, show the effect of the gaseous environment on the local LSPR generated on the nanoparticle surface, revealing selective gas adsorption sites (Fig. 2C-D). Atomic-resolution movies and time-resolved EELS are acquired to monitor the crystallographic and chemical changes in the particle during the reaction. This combined approach employed to decipher various aspects of LSPR-promoted reactions provides time-resolved, atomic-scale information on the reaction kinetics. Further our results represent a step forward in the current understanding of the dynamics of LSPR-promoted reactions and help provide insights into how to engineer nanoparticles for higher photocatalytic efficiency. [5] 


\section{References:}

[1] Sil, Devika, et al. ACS Nano 8(8) (2014), 7755-7762.

[2] Zhou, Linan, et al. Nano Lett. 16(2) (2016), 1478-1484.

[3] Thomann, Isabell, et al. Nano Lett. 11(8) (2011), 3440-3446.

[4] Nelayah, Jaysen, et al. Nature Physics, 3(5) (2007), pp.348-353.

[5] The authors acknowledge funding from the Cooperative Research agreement between the University of Maryland and the National Institute of Standards and Technology Center for Nanoscale Science and Technology, Award 70NANB14H209, through the University of Maryland.
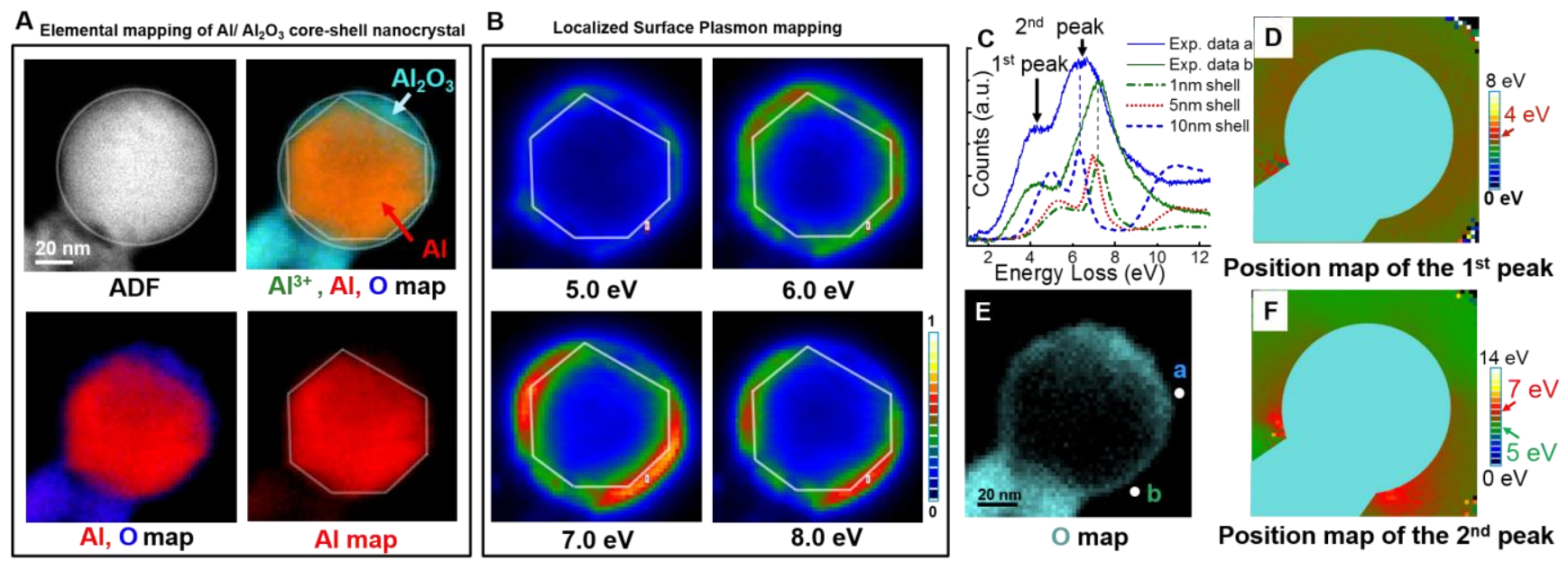

Figure 1. A, Elemental maps of the nanoparticle shows an $\mathrm{Al} / \mathrm{Al}_{2} \mathrm{O}_{3}$ core-shell structure. B, Despite the overall round shape of the particle, comparison of plasmon maps with elemental maps (A) shows correlation of LSPR intensity with the Al core crystal structure at different LSPR energies. The intensity color bar has an arbitrary unit. C, Extracted and simulated LSPR spectra show correlation between the LSPR profile and the local $\mathrm{Al}_{2} \mathrm{O}_{3}$ layer thickness. Solid lines show extracted EEL spectra from positions $\mathrm{a}$ and $\mathrm{b}$ shown in E. Dotted lines show simulated LSPR profiles of Al particles with a $70 \mathrm{~nm}$ in diameter core and a $1 \mathrm{~nm}, 5 \mathrm{~nm}$ and $10 \mathrm{~nm}$ thick shell, respectively. D-F, LSPR energy distribution of the first and second peaks (D, F) shows dependence on the thickness of $\mathrm{Al}_{2} \mathrm{O}_{3}$ shell (E) surrounding the $\mathrm{Al}$ core. The center position of the second peak (around $7 \mathrm{eV}$ ) exhibits strong correlation with the $\mathrm{Al}_{2} \mathrm{O}_{3}$ layer thickness shown in $\mathrm{E}$, while the center position of the first peak (around $4 \mathrm{eV}$ ) is not affected.
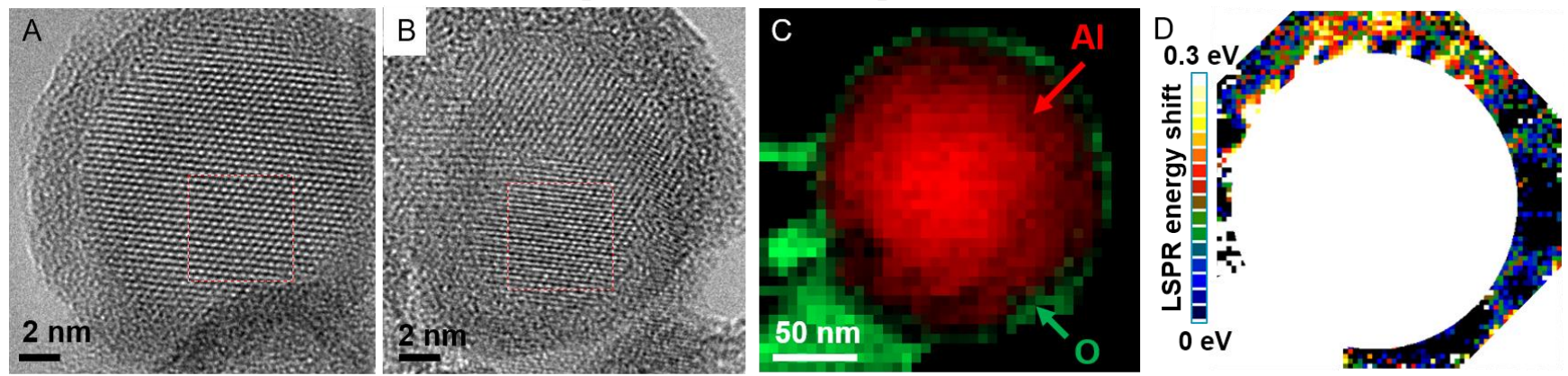

Figure 2. A-B, Snapshots, extracted from an atomic-resolution movie, showing an Al particle undergoing structural transformation in gaseous environment and under electron irradiation. C-D, Mapping of LSPR shifts (D) extracted from the LSPR maps taken before and after the gas was introduced shows preferred gas adsorption sites at the upper part of the nanoparticle, potentially due to the thinner $\mathrm{Al}_{2} \mathrm{O}_{3}$ in that region, which is demonstrated in the elemental map shown in $\mathrm{C}$. 